prevented about one case of malaria per child over the same period.

According to the WHO's 2014 world malaria report (http://www.who.int/malaria/publications/world_malaria_report_2014/en/), there were 198 million cases of malaria worldwide in 2013 and the disease killed an estimated 584,000 people. Of those deaths, $90 \%$ occurred in sub-Saharan Africa, where $83 \%$ were children under the age of 5. Extrapolating the vaccine's success to the entire region, even at the onecase-per-child rate, "that's 30 million cases of malaria prevented" every year for three or four years, Slaoui says. Given the significant burden of treating those children, the vaccine could be "an enormous benefit to public health."

As for why the vaccine works some of the time but far from all of the time, Slaoui notes the tenacity and ubiquity of the parasite. "On average, a child [in sub-Saharan Africa] gets a malaria-infected mosquito bite about once a week," he says. "You can protect against 51 of those bites over a year and fail to protect for one and the child might get malaria. It's an incredibly high hurdle."

The European regulator reviewed the vaccine under Article 58, which allows the agency to recommend a vaccine or medicine for use outside the European Union. With this endorsement in place, the WHO can now formulate a policy recommendation, and individual nations can determine how to use the vaccine-and how to fund its use-alongside existing malaria efforts. The WHO's Strategic Advisory Group of Experts on Immunization and its Malaria Policy Advisory Committee are scheduled to meet in November 2015.

A future malaria vaccine arsenal might include a so-called heterologous prime-boost approach, whereby two different vaccines containing the same antigen are given sequentially to drive a greater immune response to the parasite. Several prime-boost trials are ongoing, though a combination of RTS,S and an adenoviral vector expressing CSP from Johnson \& Johnson's Crucell vaccine division in Leiden, The Netherlands, was no more effective than Mosquirix alone, according to recently published results of a phase 2 trial (PLoS One 10, e0131571, 2015). A prime-boost strategy from a group led by Adrian Hill, director of the Jenner Institute in Oxford, UK, has shown promise in early trials using a different sporozoite antigen, ME-TRAP (multiple epitope-thrombospondin-related adhesion protein) (J. Infect. Dis. 211, 1076-1086, 2015.).

WHO spokesperson Gregory Härtl calls the vaccine a "big step forward," noting that its success will be held up as a yardstick for subsequent attempts at preventing the disease. But there's room for a single malaria vaccine with greater efficacy. "We have to find the right targets, the right antigens for $\mathrm{T}$ cells to recognize and use as a homing beacon for clearing the pathogen," says Chip Clark, CEO of Cambridge, MA-based Genocea Biosciences. Genocea's discovery efforts in malaria are supported by the Bill and Melinda Gates Foundation. The company's platform allows it to profile individuals' T-cell responses to the parasite and identify differences. With this strategy, the company hopes to find key T-cell antigens that could be added to an existing vaccine like Mosquirix or form the basis of something new. Among other approaches in early testing highlighted by PATH's Kaslow are a live, attenuated vaccine developed by Rockville, Maryland-based Sanaria that may generate a strong response to the parasite in the liver. Another strategy is a vaccine to block transmission of the parasite within the mosquito midgut.

GSK is developing a second-generation version of Mosquirix, which so far in early-stage trials in healthy volunteers is demonstrating "much higher" efficacy than what the company observed with Mosquirix at the same stage of development, says GSK's Slaoui. "It's a tweak," he says. "A highly scientific tweak with a strong rationale behind it. It's remarkable."

Chris Morrison Yardley, Pennsylvania

\title{
Around the world in a month
}

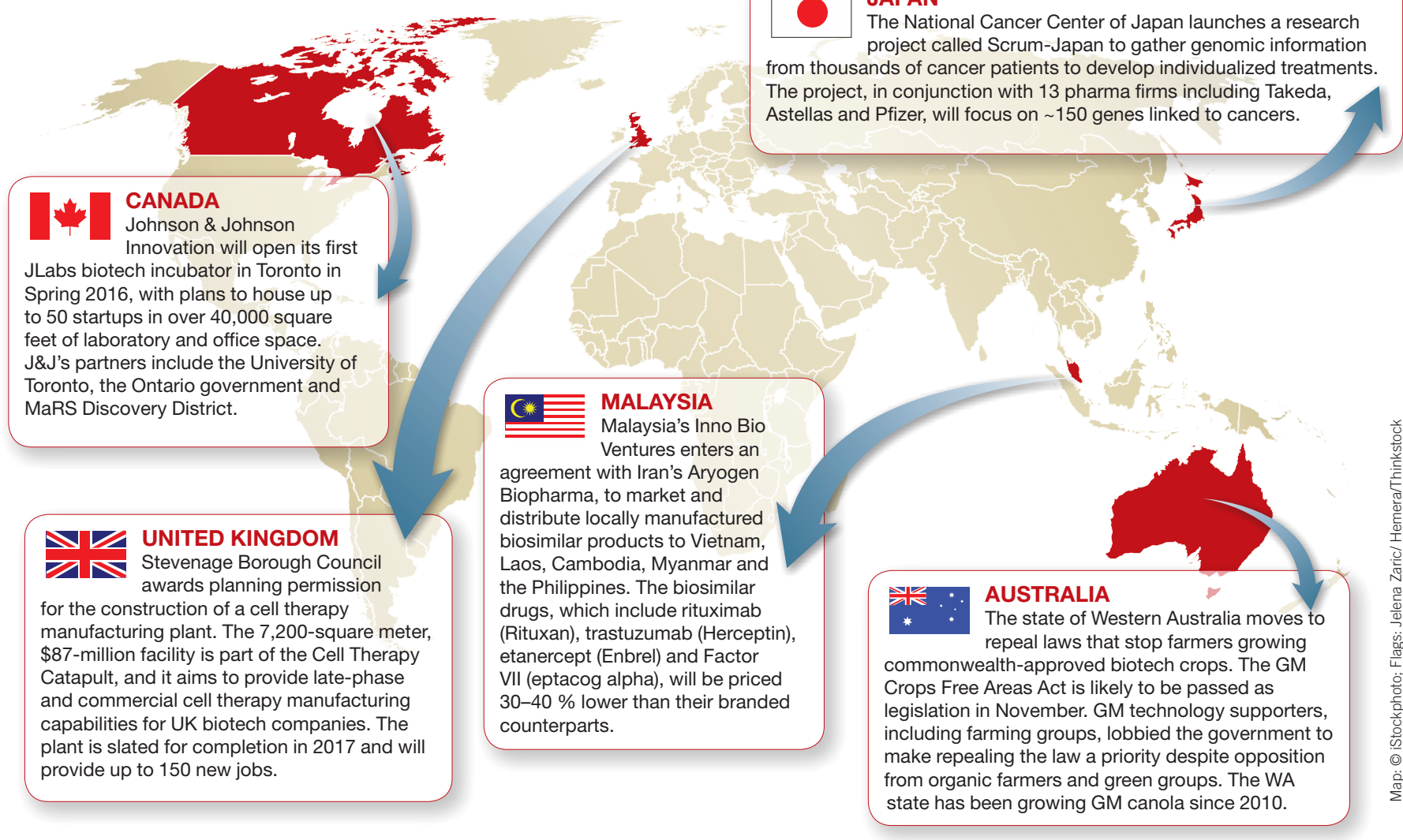

